Article

\title{
Optimization of Structure and Electrical Characteristics for Four-Layer Vertically-Stacked Horizontal Gate-All-Around Si Nanosheets Devices
}

\author{
Qingzhu Zhang ${ }^{1,2} \mathbb{D}$, Jie Gu ${ }^{1,2,3} \mathbb{D}$, Renren Xu ${ }^{1,2,3}$, Lei Cao ${ }^{1,2,3}$, Junjie Li ${ }^{1,2,3} \mathbb{D}^{\text {, Zhenhua Wu }}{ }^{1,2} \mathbb{D}$, \\ Guilei Wang ${ }^{1,2}{ }^{D}$, Jiaxin Yao ${ }^{1,2}$, Zhaohao Zhang ${ }^{1,2, *}$, Jinjuan Xiang ${ }^{1}$, Xiaobin He ${ }^{1}$, Zhenzhen Kong ${ }^{1,2}$, \\ Hong Yang ${ }^{1,2} \mathbb{D}$, Jiajia Tian ${ }^{1}$, Gaobo Xu ${ }^{1} \mathbb{D}$, Shujuan Mao ${ }^{1,2}$, Henry H. Radamson ${ }^{1}$, Huaxiang Yin ${ }^{1,2,3, *(D)}$ \\ and Jun Luo $1,2,3$
}

check for updates

Citation: Zhang, Q.; Gu, J.; Xu, R.; Cao, L.; Li, J.; Wu, Z.; Wang, G.; Yao, J.; Zhang, Z.; Xiang, J.; et al. Optimization of Structure and Electrical Characteristics for Four-Layer Vertically-Stacked Horizontal Gate-All-Around Si Nanosheets Devices. Nanomaterials 2021, 11, 646. https://doi.org/ 10.3390/nano11030646

Academic Editor: Emilio Nogales

Received: 17 January 2021

Accepted: 26 February 2021

Published: 5 March 2021

Publisher's Note: MDPI stays neutral with regard to jurisdictional claims in published maps and institutional affiliations.

\section{Copyright: (c) 2021 by the authors.} Licensee MDPI, Basel, Switzerland. This article is an open access article distributed under the terms and conditions of the Creative Commons Attribution (CC BY) license (https:// creativecommons.org/licenses/by/ $4.0 /)$.
1 Advanced Integrated Circuits R\&D Center, Institute of Microelectronic of the Chinese Academy of Sciences, Beijing 100029, China; zhangqingzhu@ime.ac.cn (Q.Z.); gujie@ime.ac.cn (J.G.); xurenren@ime.ac.cn (R.X.); caolei@ime.ac.cn (L.C.); lijunjie@ime.ac.cn (J.L.); wuzhenhua@ime.ac.cn (Z.W.); wangguilei@ime.ac.cn (G.W.); yaojiaxin@ime.ac.cn (J.Y.); xiangjinjuan@ime.ac.cn (J.X.); hexiaobin@ime.ac.cn (X.H.); kongzhenzhen@ime.ac.cn (Z.K.); yanghong@ime.ac.cn (H.Y.); tianjiajia@ime.ac.cn (J.T.); xugaobo@ime.ac.cn (G.X.); maoshujuan@ime.ac.cn (S.M.); rad@ime.ac.cn (H.H.R.); luojun@ime.ac.cn (J.L.)

2 Key Laboratory of Microelectronics Devices and Integrated Technology, Institute of Microelectronics, CAS, Beijing 100029, China

3 School of Microelectronics, University of Chinese Academy of Sciences, Beijing 100049, China

* Correspondence: zhangzhaohao@ime.ac.cn (Z.Z.); yinhuaxiang@ime.ac.cn (H.Y.); Tel.: +86-010-8299-5831 (H.Y.)

Abstract: In this paper, the optimizations of vertically-stacked horizontal gate-all-around (GAA) Si nanosheet (NS) transistors on bulk Si substrate are systemically investigated. The release process of NS channels was firstly optimized to achieve uniform device structures. An over 100:1 selective wetetch ratio of $\mathrm{GeSi}$ to $\mathrm{Si}$ layer was achieved for $\mathrm{GeSi} / \mathrm{Si}$ stacks samples with different GeSi thickness $(5 \mathrm{~nm}, 10 \mathrm{~nm}$, and $20 \mathrm{~nm})$ or annealing temperatures $\left(\leq 900^{\circ} \mathrm{C}\right)$. Furthermore, the influence of groundplane (GP) doping in Si sub-fin region to improve electrical characteristics of devices was carefully investigated by experiment and simulations. The subthreshold characteristics of $n$-type devices were greatly improved with the increase of GP doping doses. However, the $p$-type devices initially were improved and then deteriorated with the increase of GP doping doses, and they demonstrated the best electrical characteristics with the GP doping concentrations of about $1 \times 10^{18} \mathrm{~cm}^{-3}$, which was also confirmed by technical computer aided design (TCAD) simulation results. Finally, 4 stacked GAA Si NS channels with $6 \mathrm{~nm}$ in thickness and $30 \mathrm{~nm}$ in width were firstly fabricated on bulk substrate, and the performance of the stacked GAA Si NS devices achieved a larger $I_{\mathrm{ON}} / I_{\mathrm{OFF}}$ ratio $\left(3.15 \times 10^{5}\right)$ and smaller values of Subthreshold swings (SSs) $(71.2(\mathrm{~N}) / 78.7(\mathrm{P}) \mathrm{mV} / \mathrm{dec})$ and draininduced barrier lowering $(D I B L s)(9(\mathrm{~N}) / 22(\mathrm{P}) \mathrm{mV} / \mathrm{V})$ by the optimization of suppression of parasitic channels and device's structure.

Keywords: nanosheet (NS); gate-all-around (GAA); channel release; parasitic channel; suppression

\section{Introduction}

Three-dimensional (3D) fin field-effect transistors (FinFETs) have been widely used for the manufacture of high-volume integrated circuit (IC) products from $22 \mathrm{~nm}$ to $5 \mathrm{~nm}$ nodes because of their better channel electrostatic controllability and higher driving ability compared to those of conventional planar devices [1-3]. However, as the technology nodes scale down to $5 \mathrm{~nm}$ and beyond, many challenges, such as deteriorated electrostatic integrity, irresistible short-channel effects (SCEs), degraded device performance, and large process variability, appear for the FinFET structure [4,5]. Gate-all-around (GAA) Si nanowire/nanosheet (NW/NS) metal-oxide-semiconductor field effect transistors (MOSFETs) demonstrate a greater improvement in SCEs immunity than the conventional FinFETs 
due to the impact of much stronger control over the gate electrical field, and have been recognized as one of the most promising candidates beyond FinFFT technology [6-10]. In order to achieve a compatible fabrication approach with the mainstream FinFET process and improve the driving ability of the GAA NW/NS devices, stacked GAA Si NW/NS FETs have been proposed using conventional gate-last process, which provides a simple integration method by releasing NW channels from multilayer epitaxial $\mathrm{GeSi} / \mathrm{Si}$ stacks in replacement high-k dielectric/metal gate (HK/MG) trenches [11]. However, compared with the traditional bulk FinFET architecture, the fabrication of stacked GAA Si NW/NS FETs suffers from a lot of challenges, such as NSs channel release, steep fin etch, inter-diffusion restriction of $\mathrm{GeSi} / \mathrm{Si}$ stacks, inner spacers, and so on $[12,13]$. In addition, conventional techniques of parasitic sub-fin channel suppression, such as halo implantation for planar device and punchthrough stop (PTS) doping for bulk FinFET, are not suitable for GAA Si NW/NS devices, which need new approaches to reduce the leakage of parasitic sub-fin channel and improve device's subthreshold characteristics [14].

In this paper, the optimization of NS release process of stacked GAA Si NS devices was carried out and optimal GAA Si NS channels and device structure was achieved. Furthermore, the influence of ground implantation (GP) doping on the electrical characteristics of devices was systematically investigated by experiment and technical computer aided design (TCAD) simulations. Due to the optimization of the fabrication process and device characteristics, good electrical properties were achieved.

\section{Materials and Methods}

The stacked GAA Si NSs MOSFETs were fabricated on $200 \mathrm{~mm} p$-type bulk-Si (100) wafers with $8-12 \Omega \cdot \mathrm{cm}$ resistivity. The integration process flow of the device is shown in Figure 1a, which is completely compatible with conventional bulk FinFETs with a relaxed pitch. In the first place, a GP doping process with $1 \times 10^{13} \mathrm{~cm}^{-2}, 1 \times 10^{14} \mathrm{~cm}^{-2}$ and $5 \times 10^{14} \mathrm{~cm}^{-2}$ doses (NMOS: $\mathrm{B}, 140 \mathrm{keV}, 7^{\circ}$; PMOS: $\mathrm{P}, 140 \mathrm{keV}, 7^{\circ}$ ) and no implantation (the reference devices) was carried out to investigate its influence on the device's performance. In the following step, multi-layer GeSi/Si stacks with $12 \mathrm{~nm} \mathrm{Ge}_{0.3} \mathrm{Si}_{0.7}$ and $10 \mathrm{~nm} \mathrm{Si}$ were grown in a reduced pressure chemical vapor deposition (RPVVD) chamber (see Figure 1b). The patterns of fin arrays were formed by a spacer image transfer (SIT) technique $[15,16]$ with a resolution over the advanced photolithography (see Figure 1c). The vertical fin with multilayer $\mathrm{GeSi} / \mathrm{Si}$ stacks on the fin top were formed by advanced reactive ion etching (RIE) (see Figure 1d). In the following steps, a $\mathrm{SiO}_{2}$ with high aspect ratio process (HARP) was deposited, and a chemical mechanical planarization (CMP) was carried out to make the surface flat after a low temperature shallow trench isolation (STI) annealing. Then, a $\mathrm{SiO}_{2}$ etch back process was performed to reveal the fin by a diluted hydrofluoric (DHF) (see Figure 1e). A dummy gate stack with an ultra-thin oxide and thick amorphous $\mathrm{Si}(\alpha-\mathrm{Si})$ was formed on the fins, which were patterned into nanoscale gate lines with an electron beam direct writing technique (see Figure 1f). The spacers were formed by deposition of thin $\mathrm{SiN}_{x}$ and the RIE process (see Figure $1 \mathrm{~g}$ ). The $\mathrm{Ge}_{0.3} \mathrm{Si}_{0.7}$ epitaxy process with in-situ doping was carried out to reduce the parasitic resistance of SD and provide the strain to the channel (see Figure 1i). An optimized robust $\mathrm{SiN}_{x}$ ILD0 material deposited by the low pressure chemical vapor deposition (LPCVD) approach was adopted to avoid micro-trench formation and gate spacer exfoliation. Then, a global planarization process was performed by chemical mechanical planarization (CMP), and the dummy $\alpha$-Si gate lines buried in the ILD0 materials were exposed. The $\alpha$-Si dummy gate was immediately removed by immersion in the tetramethylammonium hydroxide (TMAH) (see Figure 1j). The Si NS channels were formed by a selective wet-etch of GeSi (see Figure 1k). A DHF was applied for the removal of the native oxide before the formation of a high-quality interfacial (IL) by $\mathrm{O}_{3}$. The multilayer HK/MG film stacks were deposited by atom-layer-deposition (ALD) approach on whole NSs and surrounded the NS channels to form GAA structures (see Figure 11). These complex process steps for the formation of a HK/MG by replacing the original dummy gate are together called the advanced replace metal gate (RMG) module. 
A global planarization process was performed by chemical mechanical planarization (CMP) to separate the gate. Finally, the gate and SD W-plug contact processes for the device were performed in the contact holes, and the whole device fabrication was accomplished by the formation of back-end metallization interconnection in the subsequent steps (see Figure 10).

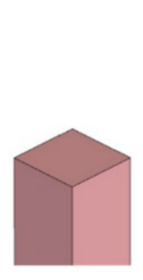

(a) PTS doping

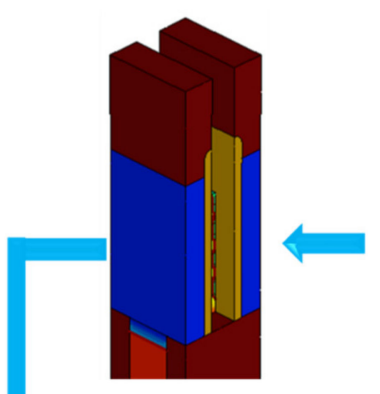

(j) Dummy gate removal

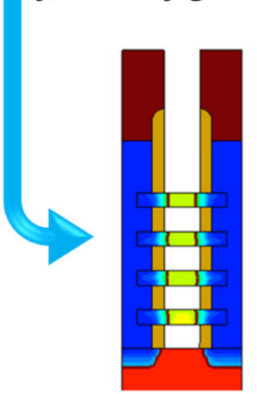

(k)NW release

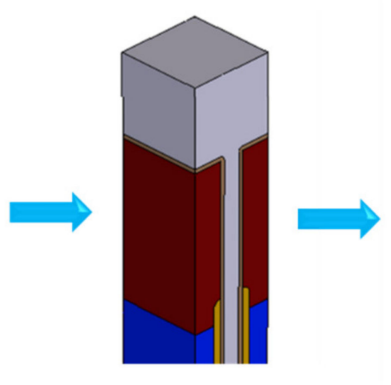

(I)HKMG (b) GeSi/Si epi

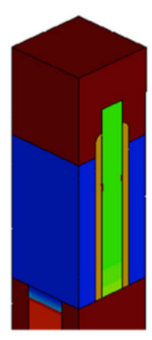

(i) ILDO

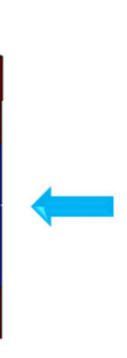

(h) SD Epi

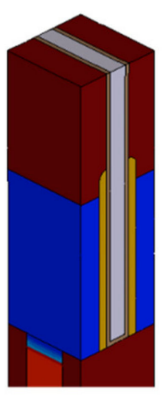

(m) CMP

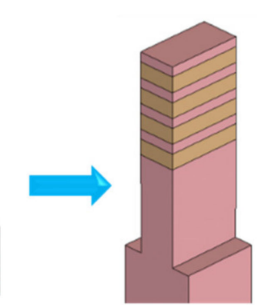

(d) Fin etch
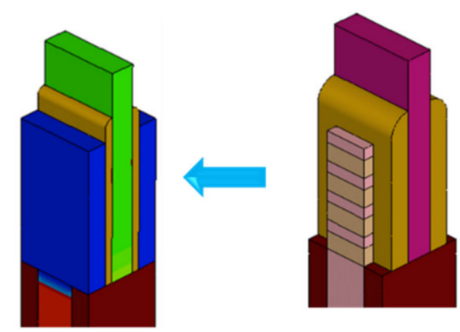

(g) spacer

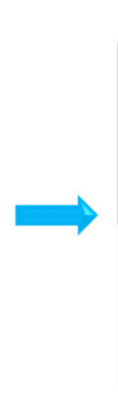

(n)ILD deposition

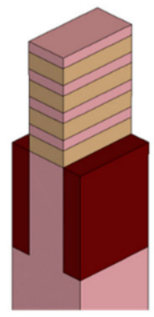

(e) STI

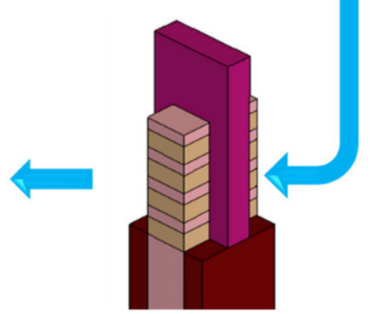

(f) Dummy gate
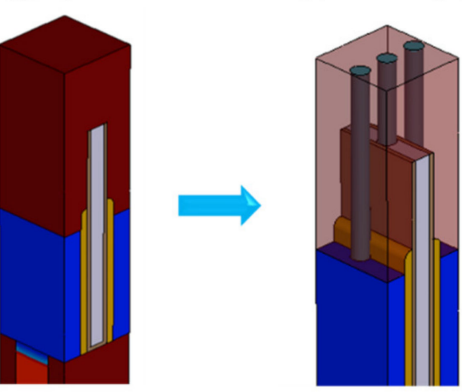

(o)Contact

Figure 1. Fabrication flow of stacked gate-all-around Si nanosheet metal-oxide-semiconductor field effect transistors (GAA Si NS MOSFET): (a) $200 \mathrm{~mm}$ p-type (100) silicon wafers after ground-plane (GP) doping, (b) epitaxy of multi-layer GeSi/Si stacks, (c) the pattern of fin arrays formed by a spacer image transfer (SIT), (d) fin etch, (e) shallow trench isolation (STI) formation, (f) dummy gate formation, (g) $\mathrm{Si}_{3} \mathrm{~N}_{4}$ spacers formation, (h) epitaxy of source drain (SD), (i) ILD0 deposition, (j) dummy gate removal, (k) Si NS channels release, (1) high-k dielectric/metal gate (HK/MG) formation, (m) chemical mechanical planarization (CMP), (n) ILD deposition, and (o) contact and metal.

For the selective etch of $\mathrm{GeSi}$ in $\mathrm{Ge}_{0.3} \mathrm{Si}_{0.7} / \mathrm{Si}$ stack structures, $20 \mathrm{~nm}$ Si layers were sandwiched between $5 \mathrm{~nm}, 10 \mathrm{~nm}$, and $20 \mathrm{~nm} \mathrm{GeSi}$ layers, and $\mathrm{Ge}_{0.3} \mathrm{Si}_{0.7} / \mathrm{Si}$ stacks in equal thickness of $20 \mathrm{~nm}$ were epitaxially grown, annealed, and analyzed. The most uniform areas on the $200 \mathrm{~mm}$ epitaxial wafers were chosen by a thickness uniformity measurement. A conventional isotropic dry etch of $\mathrm{GeSi} / \mathrm{Si}$ was carried out to form regular structures. The selected sample regions were then cut into $2 \mathrm{~cm} \times 2 \mathrm{~cm}$ slices and annealed at different rapid thermal annealing (RTA) conditions between $600{ }^{\circ} \mathrm{C}$ to $1000{ }^{\circ} \mathrm{C}$, respectively. GeSi/Si samples after different etching times in the mixed corrosion solution were laid up for $24 \mathrm{~h}$ (DHF (6\%): $\left.\mathrm{H}_{2} \mathrm{O}_{2}(30 \%): \mathrm{CH}_{3} \mathrm{COOH}(99.8 \%)=1: 2: 3\right)$.

The cross-sectional views and top views of the device's structures were observed using S-5500 and S-4800 scanning electron microscopes (SEM, Hitachi, Tokyo, Japan), respec- 
tively. The cross-sectional profiles of the final device were performed using transmission electron microscopy (TEM, FEI Talos, Brno, Czech Republic) and energy-dispersive X-ray spectroscopy (EDX, FEI Talos, Brno, Czech Republic). The electrical characterization was performed using an Agilent 4156 (Agilent, Santa Clara, USA) semiconductor parameter analyzer. The 3D TCAD simulations were carried out by using Sentaurus TCAD tools. The device model was constructed by sprocess simulation and the schematic of baseline device structure was adopted according to the real fabricated devices. Fermi-Dirac statistics as well as quantum confinement effect, Hurkx Band-to-Band model low field ballistic mobility model, Shockley-Read-Hall, and Anger recombination models were implemented in the device simulation.

\section{Results and Discussion}

\subsection{Optimization of NS Release Processes of Stacked GAA Si NS Devices}

\subsubsection{Effect of GeSi Thicknesses on NS Release in Stacked GeSi/Si Samples}

The thickness of the GeSi layer in GeSi/Si stacks determines the final spacing of stacked GAA Si NW/NS channel MOSFETs, which affects the channel morphology and the filling characteristics of HK/MG stacks $[17,18]$. In order to study the etching rate of GeSi layers and the etch selectivity of $\mathrm{GeSi}$ to $\mathrm{Si}, \mathrm{GeSi} / \mathrm{Si}$ stacks with different thicknesses of the GeSi layer ( $5 \mathrm{~nm}, 10 \mathrm{~nm}$, and $20 \mathrm{~nm}$ ) and $20 \mathrm{~nm}$ Si layer as the interlayer between $\mathrm{GeSi}$ layers were designed and fabricated. Figure 2 shows SEM images of $\mathrm{GeSi} / \mathrm{Si}$ stacks samples after different etching times ( $1 \mathrm{~min}, 3 \mathrm{~min}, 5 \mathrm{~min}, 7 \mathrm{~min}, 9 \mathrm{~min}, 11 \mathrm{~min}$, and $13 \mathrm{~min}$ ) in the mixed corrosion solution (the solution storage for $24 \mathrm{~h}$ ). As can be seen from the images, a high selective ratio of etching GeSi $(5 \mathrm{~nm}, 10 \mathrm{~nm}$, and $20 \mathrm{~nm} \mathrm{GeSi}$ in thickness) layer to $\mathrm{Si}$ layer is achieved, and the thicker the $\mathrm{GeSi}$ is, the faster the etching rate is.

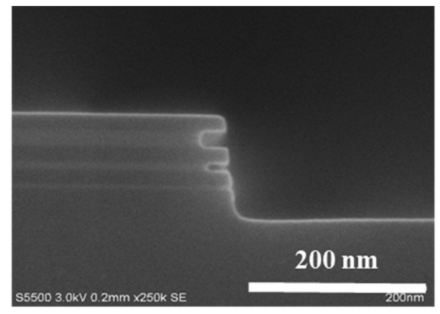

(a) 1 min

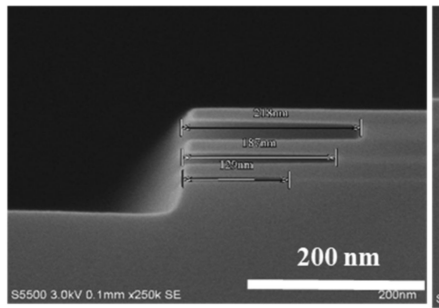

(d) $7 \mathrm{~min}$

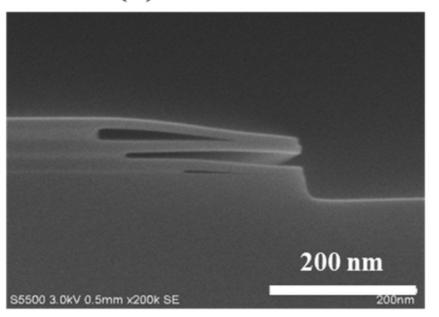

(g) 13 min

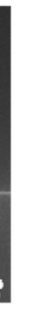

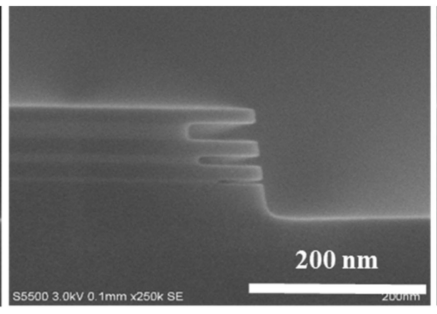

(b) $3 \mathrm{~min}$

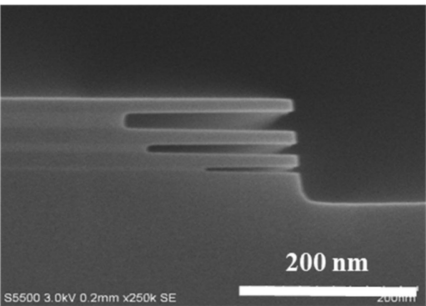

(e) $9 \mathrm{~min}$

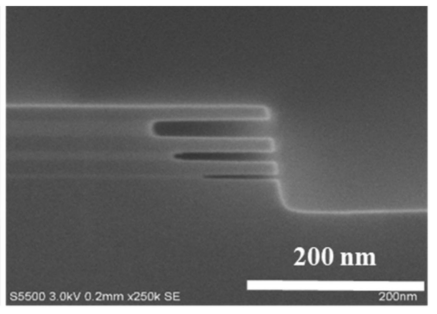

(c) $5 \mathrm{~min}$

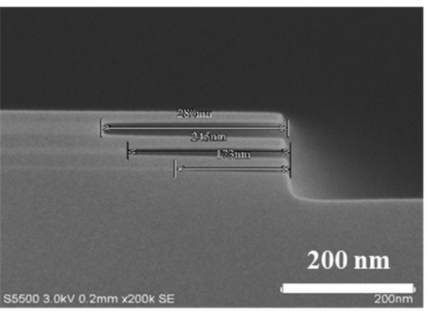

(f) $11 \mathrm{~min}$

Figure 2. Scanning electron microscopy (SEM) images of GeSi/Si stacks after different etching times: (a) $1 \mathrm{~min}$, (b) $3 \mathrm{~min}$, (c) $5 \mathrm{~min},(\mathbf{d}) 7 \mathrm{~min},(\mathbf{e}) 9 \mathrm{~min}$, (f) $11 \mathrm{~min}$, and (g) $13 \mathrm{~min}$, respectively. 
Figure 3 shows the horizontal etching lengths of $5 \mathrm{~nm}, 10 \mathrm{~nm}$, and $20 \mathrm{~nm}$ GeSi layers as a function of etching time. The etching rate of three-layer GeSi layer becomes slower with the increase of corrosion time after $7 \mathrm{~min}$, and the etching rate of thinner GeSi layer $(5 \mathrm{~nm})$ is much easier to achieve a saturate etching rate. Furthermore, the etching rate is much lower for thinner GeSi layer compared with that of thicker GeSi layer. If the etching length of GeSi layer is too long, the Si NSs are easy to "stick" together (see Figure 2g). The thinner GeSi layer may have suffered from a larger force of surface tension of liquid, so it is more likely to be an adhesion effect [10].

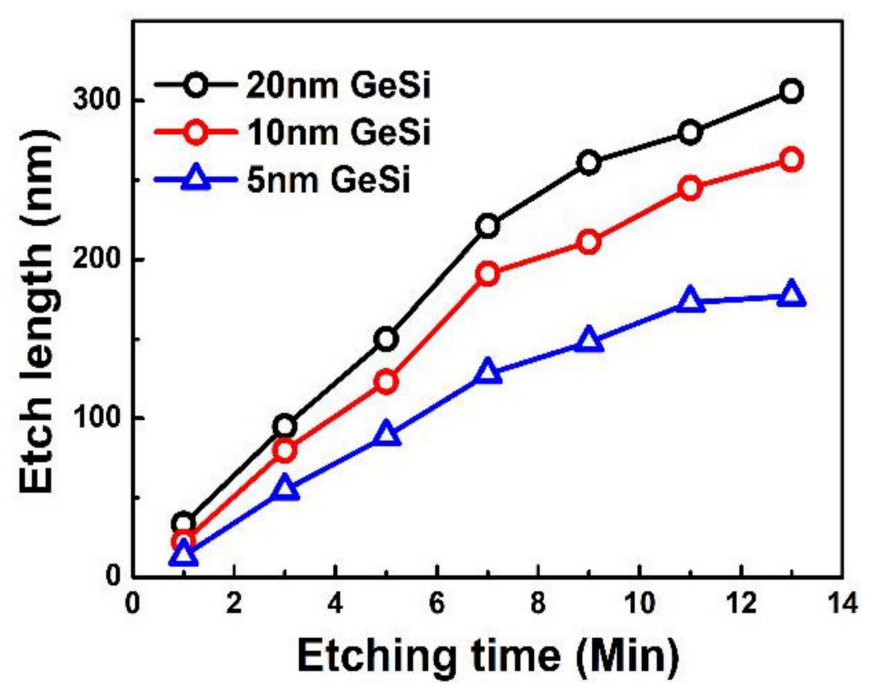

Figure 3. Etch lengths of GeSi layer as a function of etching times for GeSi/Si samples with different GeSi thicknesses.

\subsubsection{Effect of Thermal Anneal on NS Release in Stacked GeSi/Si Samples}

During the fabrication of stacked GAA Si NW/NS MOSFETs, a multi-step high temperature annealing process is needed, such as shallow trench isolation (STI) annealing and source drain (SD) activation. The high temperature processes would result in a fast atom diffusion in multi-layer $\mathrm{GeSi} / \mathrm{Si}$ stacks, and the abrupt interfaces among the multi-layer $\mathrm{GeSi} / \mathrm{Si}$ stacks layers would be destroyed, which would affect the structure, morphology, and quality of the formed Si NS channels [19-21]. In order to study the influence of annealing temperature on the $\mathrm{GeSi}$ selective etch, the samples with $\mathrm{GeSi} / \mathrm{Si}$ stack arrays were annealed at $650{ }^{\circ} \mathrm{C}, 700^{\circ} \mathrm{C}, 750{ }^{\circ} \mathrm{C}, 800^{\circ} \mathrm{C}, 850{ }^{\circ} \mathrm{C}$, and $900{ }^{\circ} \mathrm{C}$ for $30 \mathrm{~s}$, respectively, in $\mathrm{N}_{2}$ atmosphere using rapid thermal annealing (RTA) equipment. Then, all the annealed samples were put into the wet-etching solution (the solution hold on $24 \mathrm{~h}$ ) for the same $8 \mathrm{~min}$. Figure 4 shows the SEM images of the GeSi/Si samples with different annealing conditions after 8 min wet-etch. As can be seen from the images, all samples achieved high selective etching ratio of GeSi to Si below $900^{\circ} \mathrm{C}$, but the etching lengths of the GeSi layers were different.

Figure 5 shows the corresponding etching rates of the top and bottom GeSi layers. The etching rates are achieved by calculated ratio of the measured etching lengths to etching times. As can be seen from the images, the etching rate of the samples first decreases and then increases with the increase of RTA temperatures, exhibiting a "U" shape curve. The etching rate of GeSi layer is the slowest when the samples were annealing at $750{ }^{\circ} \mathrm{C}$ compared with that of other samples at higher or lower annealing temperatures. As the epitaxial growth temperature of $\mathrm{GeSi} / \mathrm{Si}$ stacks is about $725^{\circ} \mathrm{C}$, annealing at higher temperature or lower temperature processes may result in partial stress release of the $\mathrm{GeSi} / \mathrm{Si}$ stacks, which may produce defects at the $\mathrm{GeSi} / \mathrm{Si}$ interface and increase the etching rate of $\mathrm{GeSi}$ layer. The remaining $\mathrm{Si}$ layer thickness becomes thinner and the etched finger-shaped Si sheets exhibit little "warpage" at $900{ }^{\circ} \mathrm{C} / 30 \mathrm{~s}$, which may be caused by 
the inter-diffusion at $\mathrm{GeSi} / \mathrm{Si}$ interface and the stress release of the $\mathrm{GeSi} / \mathrm{Si}$ stacks, as is shown in Figure $5 f$.

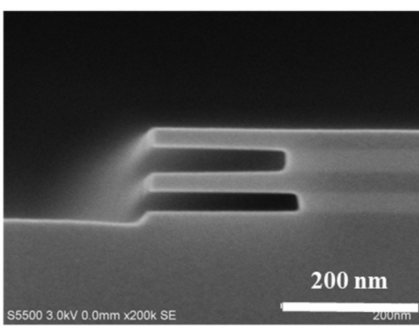

(a) $650^{\circ} \mathrm{C} / 30 \mathrm{~s}$

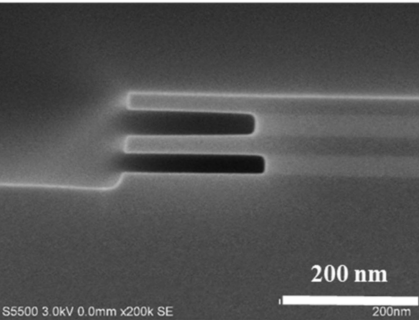

(d) $800^{\circ} \mathrm{C} / 30 \mathrm{~s}$

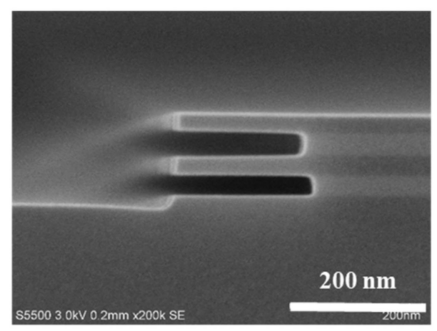

(b) $700^{\circ} \mathrm{C} / 30 \mathrm{~s}$

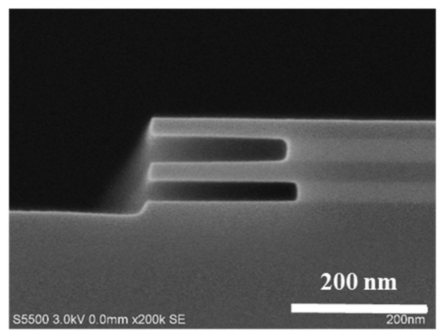

(e) $850^{\circ} \mathrm{C} / 30 \mathrm{~s}$

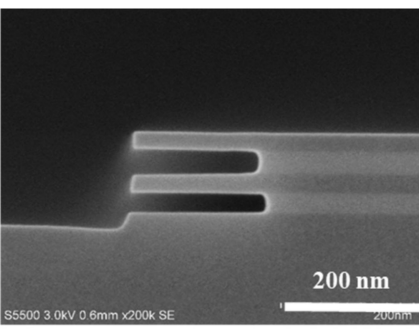

(c) $750^{\circ} \mathrm{C} / 30 \mathrm{~s}$

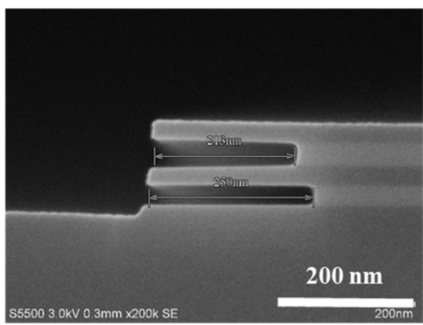

(f) $900^{\circ} \mathrm{C} / 30 \mathrm{~S}$

Figure 4. SEM images of the etched stacked $\mathrm{GeSi} / \mathrm{Si}$ samples after rapid thermal annealing (RTA) at different temperatures: (a) $650{ }^{\circ} \mathrm{C} / 30 \mathrm{~s}$, (b) $700{ }^{\circ} \mathrm{C} / 30 \mathrm{~s}$, (c) $750{ }^{\circ} \mathrm{C} / 30 \mathrm{~s}$, (d) $800{ }^{\circ} \mathrm{C} / 30 \mathrm{~s}$, (e) $850{ }^{\circ} \mathrm{C} / 30 \mathrm{~s}$, and (f) $900{ }^{\circ} \mathrm{C} / 30 \mathrm{~s}$.

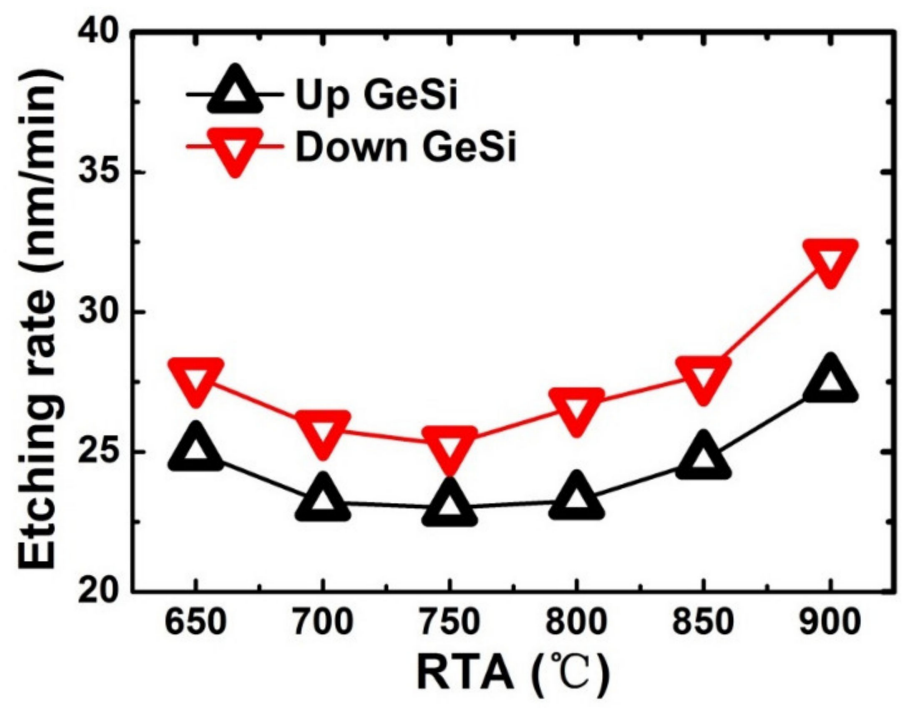

Figure 5. The etching rates of the top and bottom GeSi layers as a function of different RTA temperatures.

\subsection{Device Fabrication and Structure Characterization}

3.2.1. Process Monitoring of the Stacked Si NS Devices at Different Fabrication Stages

Figure 6 shows the SEM and TEM images of the stacked Si NS devices during different fabrication steps. Figure 6a shows the TEM image of multi-layer GeSi/Si stacks with good uniformity, and the thicknesses of GeSi and Si layers are $12 \mathrm{~nm}$ and $10 \mathrm{~nm}$, respectively. Very sharp fin with high aspect ratio is achieved by advanced dry etch process (see Figure 6b). 


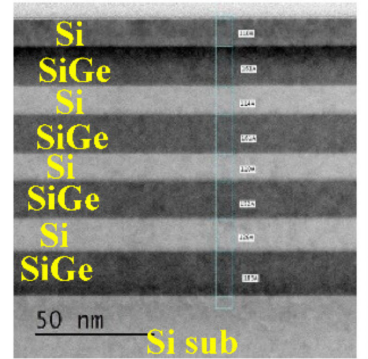

(a) Epitaxy

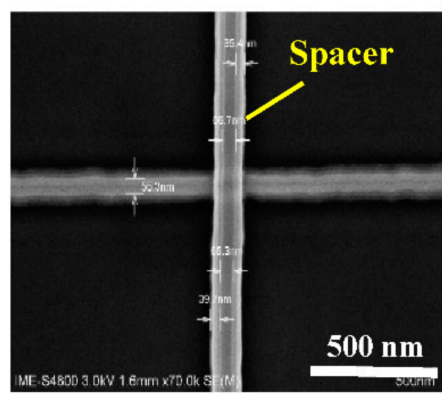

(e) Spacer

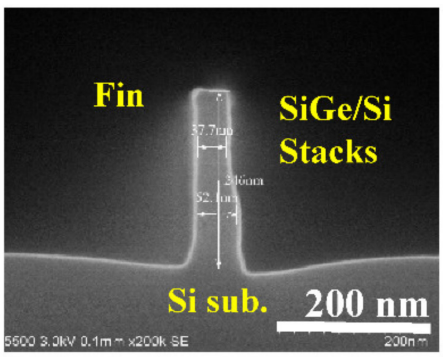

(b) Fin etch

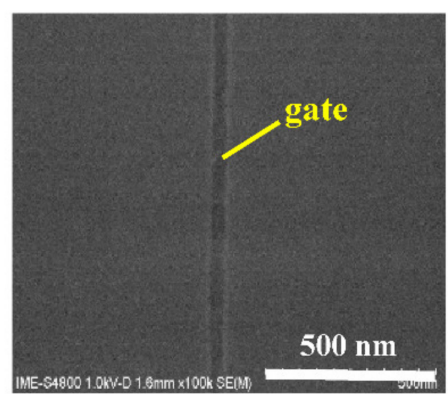

(f) CMP

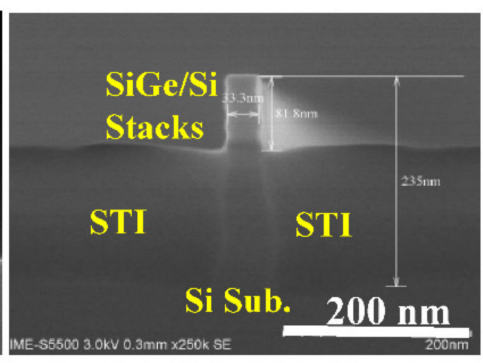

(c) STI

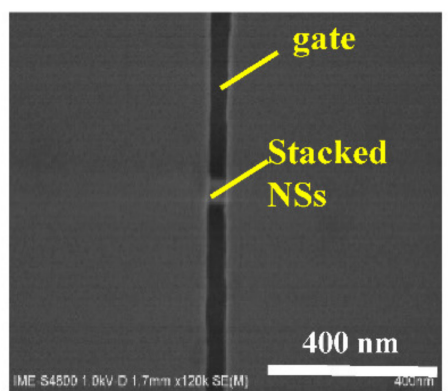

(g) Gate remove

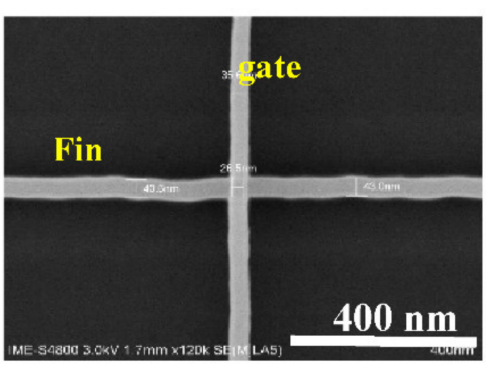

(d) Gate etch

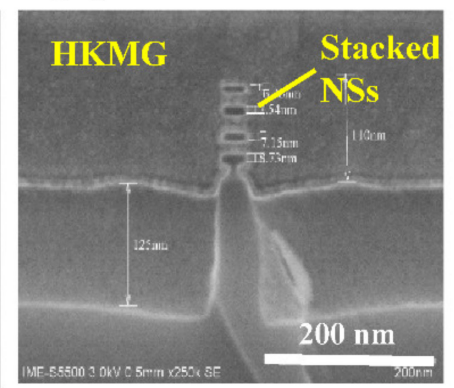

(h) HKMG

Figure 6. SEM and transmission electron microscopy (TEM) images of stacked GAA NS devices at different fabrication stages: (a) epitaxy of multi-layer GeSi/Si stacks, (b) fin etch by reactive ion etching (RIE) process, (c) STI formation, (d) dummy gate formation, (e) spacer formation, (f) poly gate open by CMP, (g) dummy gate removal, and (h) Si NS release and HK/MG fill.

Figure 6c shows the cross-sectional SEM image of the device after shallow trench isolation (STI) formation. As can be seen from the images, the STI is well controlled with very flat surfaces and the multilayer $\mathrm{GeSi} / \mathrm{Si}$ stacks on the top of the fin are revealed. Figure $6 \mathrm{~d}$ shows the top view SEM image of devices after gate formation, which is $\sim 30 \mathrm{~nm}$ in width. Conformal spacers are observed at two sides of dummy gate, which are used as self-aligned mask for SD doping (see Figure 6e). A top view of stacked GAA Si NS devices after dummy gate CMP is shown in Figure $6 \mathrm{f}$. The surface of ILD0 layer is relatively smooth, and a $\sim 30 \mathrm{~nm}$ poly gate is revealed. Figure $6 \mathrm{~g}$ shows a top view of the $30-\mathrm{nm}-L_{\mathrm{g}}$ device after dummy gate removal and Si NSs release. The $\alpha$-Si dummy gate was entirely removed, and the stacked Si NS channels appear in the open gate trench. Figure 6h shows the cross-sectional SEM image of the stacked GAA Si NS channels after deposition of multilayer ALD HK/MG stacks. As can been seen from the image, very uniform 4-layer stacked NS channels are formed and well surrounded by HK/MG stacks.

\subsubsection{Structure Characterization of the Stacked GAA Si NS Devices}

Figure 7a shows a schematic of the stacked GAA Si NSs device, and Figure 7b,c are cross-sectional TEM images along different directions ( $\mathrm{AA}^{\prime}$ cut across the fin top and BB' cut across the channel direction) and corresponding EDX maps for O, N, Si, Hf, Ti, and W distribution, respectively. As can be seen from Figure $7 \mathrm{~b}$, the physical $L_{\mathrm{g}}$ is $25.8 \mathrm{~nm}$ and the stacked GAA Si NSs device is well fabricated, because the SD fin, spacer, and gate trench are well protected with conformal ILD0 material, allowing the Si NS channels and conformal HK/MG GAA structure to be preserved after the final process steps. As can be seen from Figure 7c, there are four uniform stacked Si NS channels formed and thickness of the NSs is about $6 \mathrm{~nm}$, implying the well-controlled Si NS release and fabrication processes [22-24]. The Si NSs channel were surrounded by the conformal ALD multilayer HKMG stacks to form GAA structure, which could provide a good gate control ability to the ultrathin Si NS channels. However, there is residual of the bottom GeSi layer for stacked GAA Si 
NS channel, which deteriorates the gate control ability and electrical characteristics of the devices [25].

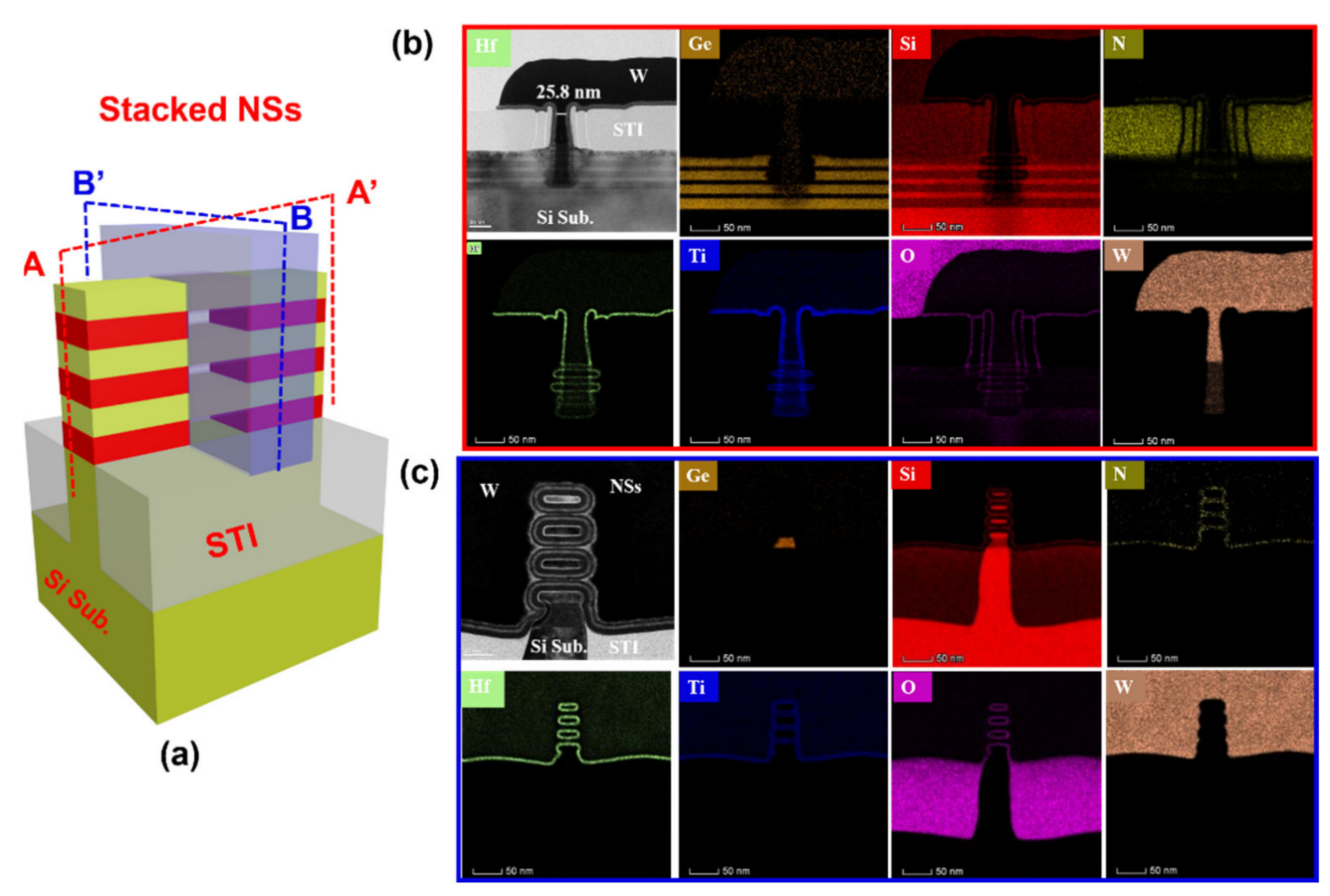

Figure 7. (a) A schematic of the stacked GAA Si NSs device, and $(\mathbf{b}, \mathbf{c})$ show cross-sectional TEM images along the direction of $\mathrm{AA}^{\prime}$ (cut across the fin top) and BB' (cut across the channel direction), and the corresponding energy-dispersive $\mathrm{X}$-ray spectroscopy (EDX) maps for Ge, Si, N, Hf, Ti, O and W distribution, respectively.

\subsubsection{Si NS Channels Release Control with Different Etching Processes}

In the actual fabrication of the stacked GAA Si NS devices, there is the complex integration approach, variation of process fluctuation, and Si NS channels releasing process control, resulting in the non-ideal stacked GAA Si NS channels $[26,27]$. In the NSs release processes of stacked Si NS channels, the NS channels are easy to stick to each other, especially in the case of wet etching approach with a large interfacial tension, as shown in Figure 8a. As can be seen from the image, the upper two NSs are connected together, and the bottom two NSs are also connected together, which affects the filling of multi-layer HK/MG stacks and the gate control characteristics of the devices. Figure $8 \mathrm{~b}$ shows the cross-sectional TEM images of stacked GAA Si NS channels with the residual of the bottom $\mathrm{GeSi}$, which may be caused by a higher height of the STI preventing the bottom GeSi from etching. The non-ideal structure with the residual of the bottom $\mathrm{GeSi}$ introduces the parasitic channels at the bottom of the channel and deteriorates the gate control ability of the devices [27]. There is also the phenomenon of NS channels sticking to each other and to the residual of the bottom GeSi in the NSs channels, which greatly degrades the performance of the devices, as shown in Figure $8 \mathrm{c}$. Figure $8 \mathrm{~d}$ shows the cross-sectional TEM images of stacked GAA Si NS channels with the complete NS release by controlling the Si NS release process. As can be seen from image, the device's structure without the residual bottom GeSi could form the ideal GAA Si NSs structure with conformal ALD HK/MG stacks. 

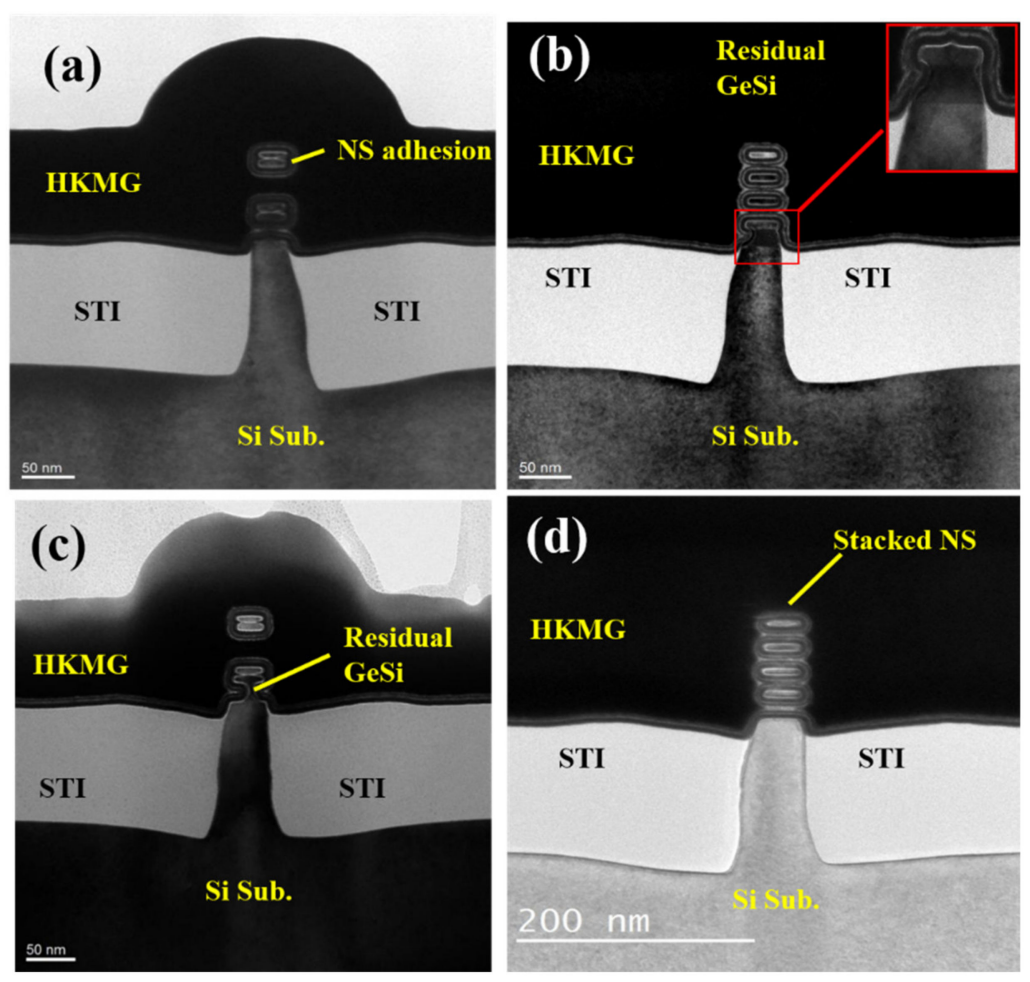

Figure 8. Cross-sectional TEM images of stacked GAA Si NS channel with (a) NS channels sticking to each other, (b) the residual of the bottom GeSi, (c) NSs channels sticking to each other and a thin residual of the bottom $\mathrm{GeSi}$, and (d) complete NS release.

\subsection{Influence of GP Doping on Performance of Devices \\ 3.3.1. Experimental Results of GP Doping on Performance of Devices}

In this part, an effective approach of the GP doping was selected to suppress parasitic sub-fin channels of the 4-layer stacked GAA Si NSs devices based on FinFET fabrication flow. The simulated doping concentrations as a function of the $\mathrm{P}$ and $\mathrm{B}$ doping doses $\left(1 \times 10^{13} \mathrm{~cm}^{-2}, 1 \times 10^{14} \mathrm{~cm}^{-2}\right.$, and $\left.5 \times 10^{14} \mathrm{~cm}^{-2}\right)$ are shown in Figure 9a. There is approximately a linear relationship between doping concentrations and doses, and the $\mathrm{P}(\mathrm{B})$ doping concentrations are approximately $6 \times 10^{16}\left(9 \times 10^{16}\right) \mathrm{cm}^{-3}, 7 \times 10^{17}\left(8 \times 10^{17}\right) \mathrm{cm}^{-3}$, and $3 \times 10^{18}\left(4 \times 10^{18}\right) \mathrm{cm}^{-3}$, respectively. Figure $9 \mathrm{~b}, \mathrm{c}$ show the typical experimental transfer curves of $n$-/ $p$-type stacked GAA Si NSs devices with no implantation, $1 \times 10^{13} \mathrm{~cm}^{-2}$ and $5 \times 10^{14} \mathrm{~cm}^{-2}$, respectively. As can be seen from the images, the drain currents are almost the same at on-state, but there is a great influence on the leakages at off-state both for $n$-type and $p$-type stacked GAA Si NSs devices, respectively. The leakages are reduced by approximately two orders of magnitude for $n$-type GAA Si NSs device by using a GP doping $\mathrm{P}$ dose of $1 \times 10^{13} \mathrm{~cm}^{-2}$. However, there are approximately over three orders of magnitude for $p$-type with a GP doping B dose of $1 \times 10^{13} \mathrm{~cm}^{-2}$. There are approximately another two orders of magnitude reduction in leakages for $n$-type with a GP doping dose of $5 \times 10^{14} \mathrm{~cm}^{-2} \mathrm{P}$ compared to those with $1 \times 10^{13} \mathrm{~cm}^{-2}$ (see Figure 9c). However, the leakage increases approximately two orders of magnitude for $p$-type with a GP doping dose of $5 \times 10^{14} \mathrm{~cm}^{-2} \mathrm{~B}$ compared to those with $1 \times 10^{13} \mathrm{~cm}^{-2}$ (see Figure $9 \mathrm{~b}$ ). The values of $I_{\mathrm{ON}} / I_{\mathrm{OFF}}$ ratios of $S S$ s are extracted from the transfer curves with different GP doping conditions, as is shown in Figure $9 \mathrm{~d}$,e, respectively. The $I_{\mathrm{ON}} / I_{\mathrm{OFF}}$ ratios of $n$-type devices increase with the increase of GP doping doses. However, the $I_{\mathrm{ON}} / I_{\mathrm{OFF}}$ ratios of $p$-type devices exhibit first increases and then decreases with the increase of GP doping doses. Meanwhile, the values of $S S_{\text {lin }}$ and $S S_{\text {sat }}$ decrease with the increase of GP doping doses for $n$-type devices, but the $p$-type devices also exhibit a trend of faster decreases and then increases with the increase of GP concentrations (see Figure 9e). The results show that the $n$-type and $p$-type stacked GAA Si NSs devices need different GP doping conditions, 
and the optimization of parasitic channel for $p$-type devices is more difficult and complex than that of $n$-type devices. The $I_{\mathrm{ON}}-I_{\mathrm{OFF}}$ mapping of $n$-type stacked GAA Si NS devices with different GP doping conditions is shown in Figure 9 f. The current density of $I_{\mathrm{ON}}$ and $I_{\mathrm{OFF}}$ is normalized according to the width of the Si nanosheets. As can been seen from the images, the off-current of the device is reduced by four orders of magnitude compared with that of without GP doping.
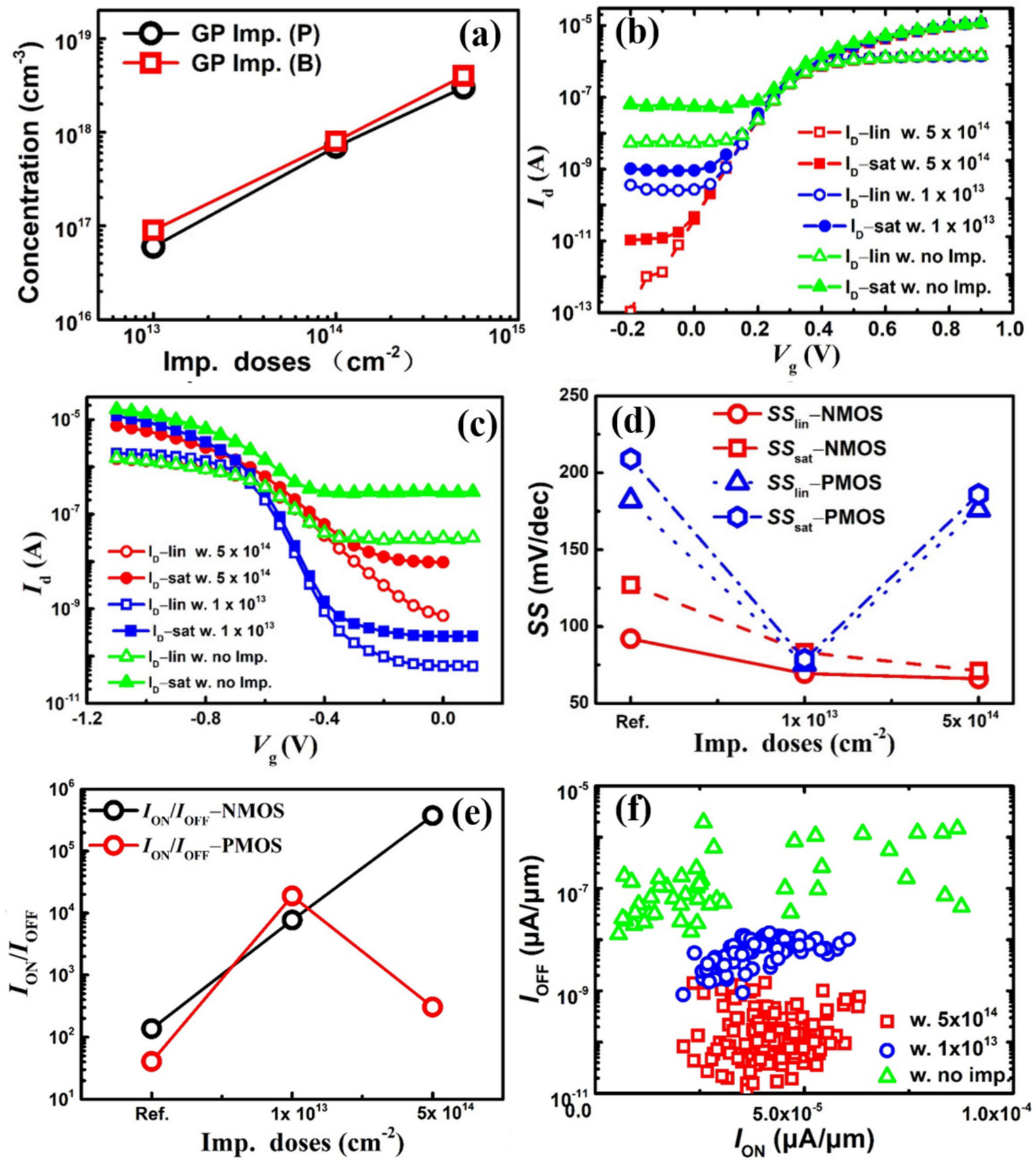

Figure 9. (a) Concentration of dopants as a function of doping doses of implant doping doses, (b,c) experimental $I_{\mathrm{D}}-V_{\mathrm{G}}$ of $n$ - $/ p$-type stacked GAA Si NS devices, $(\mathbf{d}, \mathbf{e}) I_{\mathrm{ON}} / I_{\mathrm{OFF}}$ ratios, $S S_{\text {lin }}$ and $S S_{\text {sat }}$ extracted with different GP doping conditions, and (f) $I_{\mathrm{ON}}-I_{\mathrm{OFF}}$ mapping of $n$-type stacked GAA Si NSs devices. 


\subsubsection{Simulation Results of GP Doping on Performance of $p$-Type Devices}

In order to further study the influence of GP doping on the suppression of parasitic channels and seek out the optimal conditions for $p$-type stacked GAA Si NSs devices application, 3D TCAD simulations on the stacked GAA Si NSs devices, including process and device simulations, were carried out using Sentaurus TCAD tools. Figure 10 shows the schematic of device structure adopted in this paper similar to the real structure of the fabricated devices, and the stacked GAA Si NS device comprises 4 stacked NSs channels (see Figure 10b,c). Table 1 summarizes the parameters used in the simulation, and ultra-scaled $2 \mathrm{~nm}$ node device with 12-nm- $L_{\mathrm{g}}$ and 4-layer stacked 6-nm-thick Si NSs channels were adopted according to the International Roadmap for Devices and Systems (IRDS) [28]. Hurkx Band-to-Band model as well as Fermi-Dirac statistics, Shockley-ReadHall, and Anger recombination models were implemented in the device simulation [29].

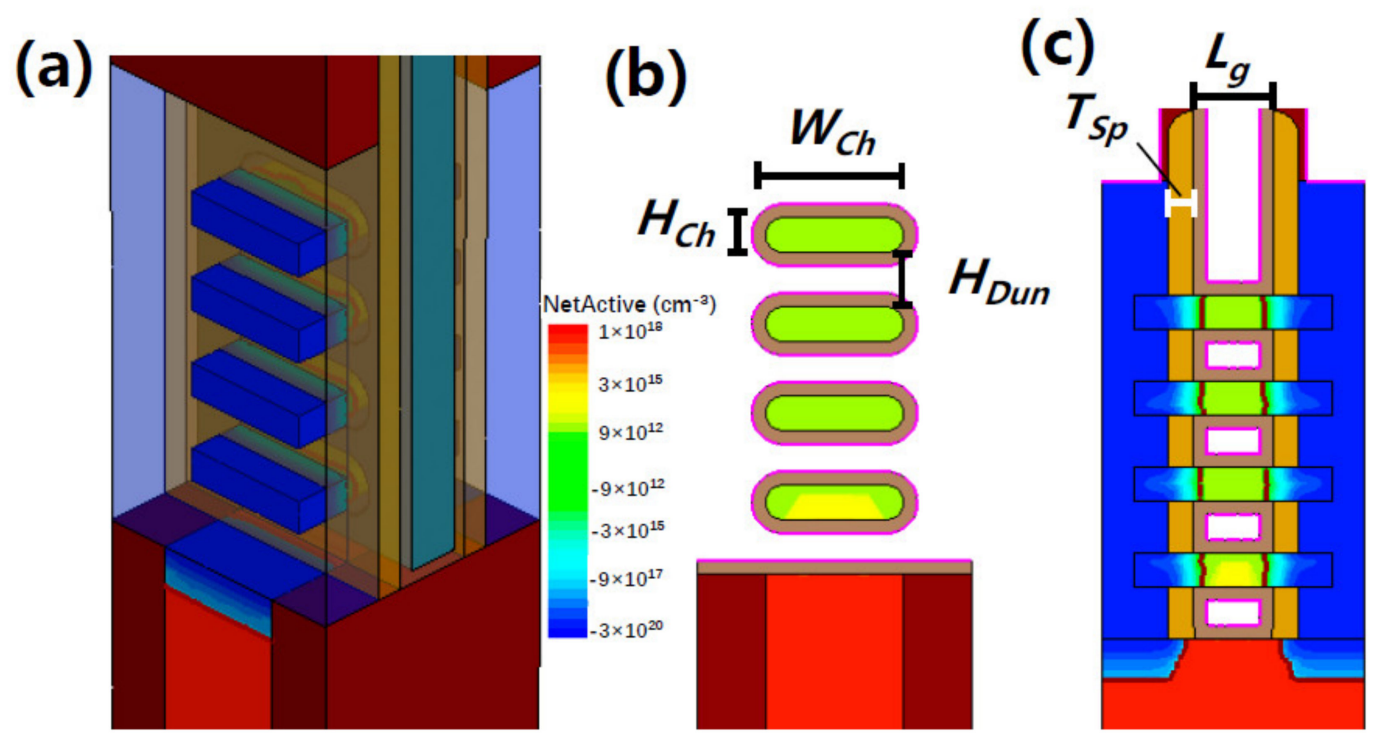

Figure 10. (a) Simulated GAA Si NS device's structure and cross-sectional images (b) perpendicular to and (c) along the channel direction.

Table 1. Parameters defined in stacked GAA Si NS device.

\begin{tabular}{cc}
\hline Parameters of GAA Si NS Devices & Values \\
\hline Type & $p$-type \\
Stacks of NS channels & 4 \\
$L_{\mathrm{g}}(\mathrm{nm})$ & 12 \\
NS thickness (nm) & 5 \\
Wideness of NSs (nm) & 20 \\
EOT (nm) & 1 \\
Spacing between NSs (nm) & $8 \mathrm{~nm}$ \\
$\mathrm{~N}_{\mathrm{ch}}\left(\mathrm{cm}^{-3}\right)$ & $1 \times 10^{15}$ \\
$\mathrm{~N}_{\mathrm{SD}}\left(\mathrm{cm}^{-3}\right)$ & $3 \times 10^{20}$ \\
Thickness of spacers $(\mathrm{nm})$ & $4 \mathrm{~nm}$ \\
\hline
\end{tabular}

The transfer curves of 12-nm- $\mathrm{g}_{\mathrm{g}}$-type stacked GAA Si NSs devices with different GP concentrations are shown in Figure 11a. 

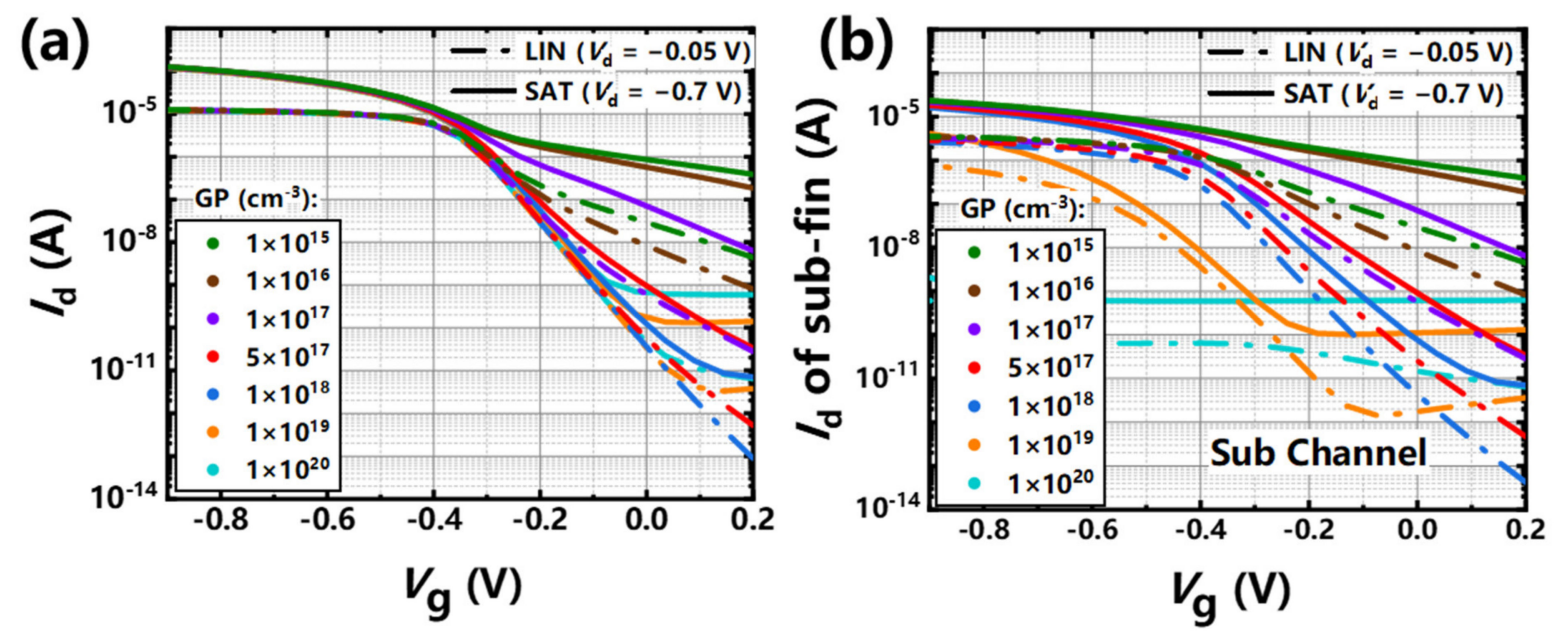

Figure 11. $I_{\mathrm{d}}-V_{\mathrm{g}}$ curves of $12-\mathrm{nm}-\mathrm{Lg}$, (a) NS-FETs and (b) sub-channel with different GP doping concentrations.

As can be seen from the image, there are large leakages at a GP doping concentration of $3 \times 10^{18} \mathrm{~cm}^{-3}$ and the leakages get smaller with the increase of GP doping concentrations. However, the leakage becomes more serious and exhibits obvious gate-induced drain leakage (GIDL) effect when the GP concentration reach $1 \times 10^{19} \mathrm{~cm}^{-3}$. In order to study and analyze the detailed reasons of the phenomenon, the corresponding changes of $I_{\mathrm{d}}-V_{\mathrm{g}}$ curves of the parasitic sub-fin (by excluding the current of the NS channels) under different GP concentrations are shown in Figure 11b. The leakage current of parasitic sub-fin channel at the bottom shows two trends with the increase of doping concentrations: (1) fast decrease of the off-state current; and (2) shift of the $V_{\text {th }}$ to the negative direction. The leakage of the sub-fin at off-state has no obvious change with the decrease of $V_{\mathrm{g}}$ with the GP doping dose of $1 \times 10^{15} \mathrm{~cm}^{-2}$, which is caused by a large punch through current from sub-fin and deteriorates the device's performance. With the increase of GP doping concentrations, the leakage current of the sub-fin at the off-state decreases exponentially and the values of $V_{\mathrm{t}}$ shift become a little larger, which is more helpful to reduce the off-state leakage of the whole device. There is a large $V_{\mathrm{t}}$ shift $(\sim 200 \mathrm{mV})$ of the $I_{\mathrm{d}}-V_{\mathrm{g}}$ curves of the sub-fin at GP doping concentration of $1 \times 10^{19} \mathrm{~cm}^{-3}$ compared with that of with a GP doping concentration of $1 \times 10^{18} \mathrm{~cm}^{-3}$. However, the off-state leakage of the sub-fin gets larger and the curves exhibit gate induced barrier lowering (GIDL), which may be caused by a larger overlap for $p$-type devices. The leakage current of the sub-fin remains stable and does not change with the increase of $V_{\mathrm{g}} \mathrm{s}$ with a GP doping concentration of $1 \times 10^{20} \mathrm{~cm}^{-3}$, which demonstrates another side-effect of band-to-band tunneling (BTBT) current occurring in degenerate semiconductor $p-n$ junction at very high GP doping concentrations.

In order to further investigate the influence of sub-fin channels with different GP doping concentrations on the performance of stacked GAA Si NSs devices with different $L_{\mathrm{g}} \mathrm{s}$, some key electrical parameters of the devices (e.g., DIBLs, $S S_{\mathrm{sat}} \mathrm{s}, I_{\mathrm{OFF}}$, and $I_{\mathrm{ON}} / I_{\mathrm{OFF}}$ ratios) are extracted and shown in Figure 12.

The current density of the $I_{\mathrm{OFF}}$ is normalized according to the width of the stacked Si nanosheets. The values of DIBLs and $S S_{\text {sat }}$ s as a function of different GP doping concentrations with different $L_{\mathrm{g}}$ s for the stacked GAA Si NS device are shown in Figure 12a. The values of DIBLs and $S S_{\text {sat }}$ s have a fast initial decrease with the increase of GP doping concentrations and almost remain stable when GP doping concentrations are over $5 \times 10^{17} \mathrm{~cm}^{-3}$. Meanwhile, the values of DIBLs and $S S_{\text {sat }}$ increase with the decrease of $L_{\mathrm{g} S}$ and the deteriorated DIBLs and $S S_{\text {sat }}$ s could also be improved even when the $L_{\mathrm{g}} \mathrm{s}$ is scaled to $12 \mathrm{~nm}$ for stacked GAA Si NS devices. In addition, the minimum $I_{\mathrm{OFF}} \mathrm{S}$ and the largest $I_{\mathrm{ON}} / I_{\mathrm{OFF}}$ ratios are achieved at GP doping concentrations of $1 \times 10^{18} \mathrm{~cm}^{-3}$ for different $L_{\mathrm{g}} \mathrm{S}$ and these parameters worsen because of the induced tunneling current for the degenerated semiconductor effect (Figure 12b,c). Furthermore, the electrical conductance in bottom 
NS channel is degraded for high-concentration-dopant out-diffusion phenomena in this technology. Therefore, the GP doping doses of approximately $1 \times 10^{14} \mathrm{~cm}^{-2}$ is a better condition for reducing sub threshold leakage and improving $p$-type device performance.
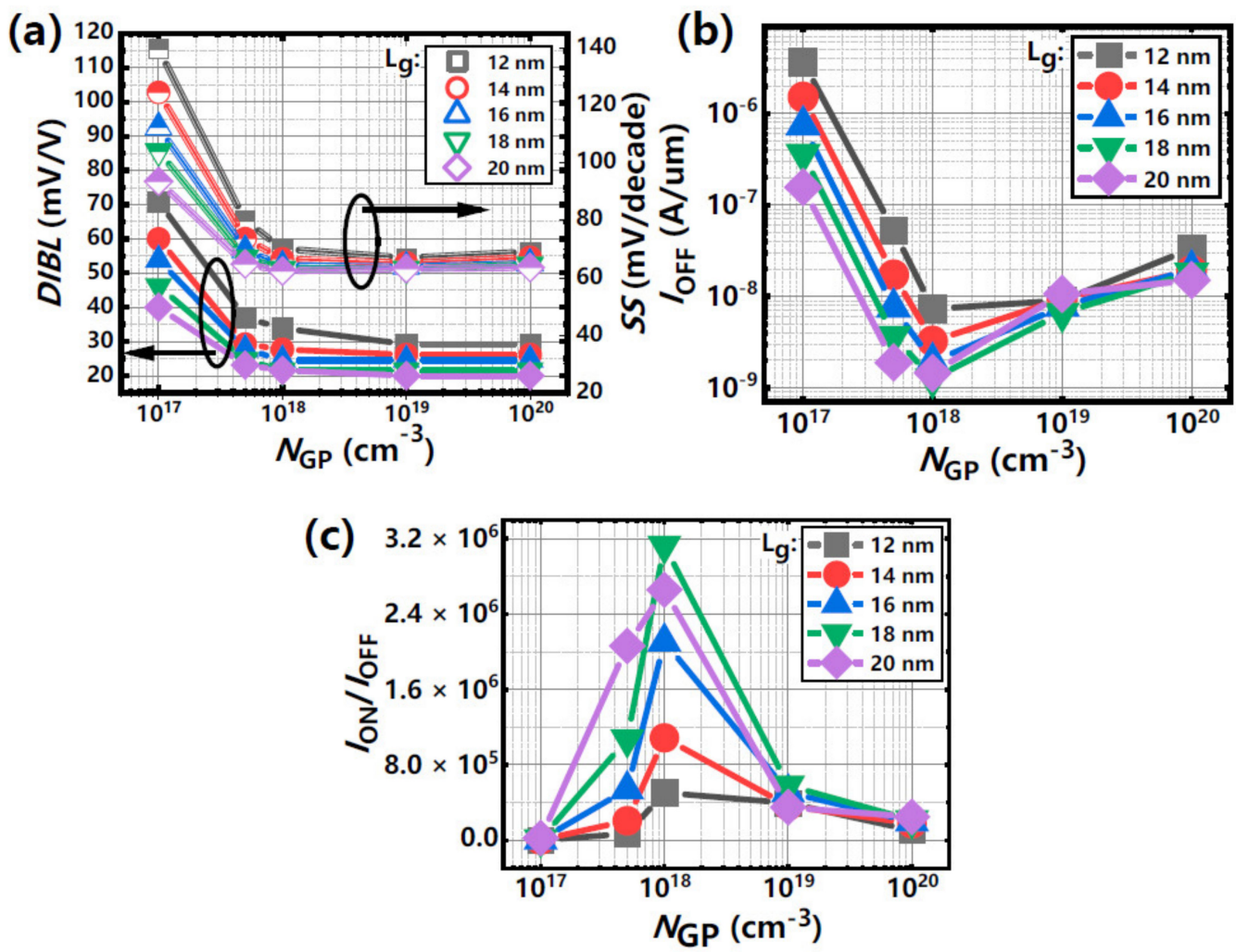

Figure 12. (a) DIBL and $S S_{\mathrm{sat}}$, (b) $I_{\mathrm{OFF}}$ and (c) $I_{\mathrm{ON}} / I_{\mathrm{OFF}}$ ratio extracted from devices with different $L_{\mathrm{g} S}$ and GP doping concentration.

Table 2 shows a system parameters comparison of some relevant reported results in recent years and our fabricated stacked GAA Si NS devices. For the first time, 4layer stacked GAA Si NS channels with $6 \mathrm{~nm}$ in thickness and $30 \mathrm{~nm}$ in width were firstly fabricated on bulk substrate and achieved good morphology of $n$ - $/ p$-type GAA Si NS devices. Furthermore, a larger $I_{\mathrm{ON}} / I_{\mathrm{OFF}}$ ratio and smaller values of $S S$ s and DIBLs for $25.8 \mathrm{~nm}-L_{\mathrm{g}}$ devices is achieved for the fabricated stacked GAA Si NS devices by the optimization of suppression of parasitic channels and device's structure. The results indicated that the stacked GAA Si NS devices fabricated have much better comprehensive characteristics compared with those of the compared devices reported [10-13,30].

Table 2. A comparison of GAA Si NS devices in recent years.

\begin{tabular}{|c|c|c|c|c|c|c|}
\hline Ref. & $\begin{array}{c}\text { (30 IBM) } \\
2017 \text { VLSI }\end{array}$ & $\begin{array}{l}\text { (11 Samsung) } \\
2018 \text { IEDM }\end{array}$ & $\begin{array}{l}\text { (12 NNDL) } \\
2018 \text { EDL }\end{array}$ & $\begin{array}{c}\text { (13 IBM) } \\
2019 \text { IEDM }\end{array}$ & $\begin{array}{l}\text { (14 CEA Leti) } \\
2020 \text { VLSI }\end{array}$ & $\begin{array}{l}\text { (IMECAS) } \\
\text { This Work }\end{array}$ \\
\hline Channel & Stacked Si NS & Stacked Si NS & Stacked Ge NS & Stacked Si NS & Stacked Si NS & Stacked Si NS \\
\hline N/P type & $\mathrm{N} / \mathrm{P}$ & $\mathrm{N} / \mathrm{P}$ & $\mathrm{N} / \mathrm{P}$ & $\mathrm{N} / \mathrm{P}$ & $\mathrm{N}$ & $\mathrm{N} / \mathrm{P}$ \\
\hline Fin/Sub. & $\begin{array}{c}\mathrm{GeSi} / \mathrm{Si} \text { stack } \\
\text { on } \mathrm{Si}\end{array}$ & - & $\begin{array}{c}\mathrm{Ge} / \mathrm{Si} \text { stack on } \\
\text { SOI }\end{array}$ & $\begin{array}{c}\mathrm{SiGe} / \mathrm{Si} \text { stack } \\
\text { on } \mathrm{Si}\end{array}$ & $\begin{array}{c}\mathrm{SiGe} / \mathrm{Si} \text { stack } \\
\text { on SOI }\end{array}$ & $\begin{array}{c}\mathrm{SiGe} / \mathrm{Si} \text { stack } \\
\text { on } \mathrm{Si}\end{array}$ \\
\hline $\begin{array}{c}\text { Num. } \\
\text { of Stacked NSs }\end{array}$ & 3 & - & 3 & 3 & 7 & 4 \\
\hline STI Annael & $\leq 900{ }^{\circ} \mathrm{C}$ & - & - & $\leq 900^{\circ} \mathrm{C}$ & - & $\leq 850{ }^{\circ} \mathrm{C}$ \\
\hline
\end{tabular}


Table 2. Cont.

\begin{tabular}{ccccccc}
\hline Ref. & $\begin{array}{c}\text { (30 IBM) } \\
\text { 2017 VLSI }\end{array}$ & $\begin{array}{c}\text { (11 Samsung) } \\
\text { 2018 IEDM }\end{array}$ & $\begin{array}{c}\text { (12 NNDL) } \\
\text { 2018 EDL }\end{array}$ & $\begin{array}{c}\text { (13 IBM) } \\
\text { 2019 IEDM }\end{array}$ & $\begin{array}{c}\text { (14 CEA Leti) } \\
\text { 2020 VLSI }\end{array}$ & $\begin{array}{c}\text { (IMECAS) } \\
\text { This Work }\end{array}$ \\
\hline Release & In RMG & In RMG & In RMG & In RMG & In RMG & In RMG \\
\hline NS forming & SiGe etch & - & Si etch & SiGe etch & SiGe etch & SiGe etch \\
\hline $\begin{array}{c}\text { NS } \\
\text { Width/Thickness }\end{array}$ & $\sim 25 / 6 \mathrm{~nm}$ & - & $\sim 90 / 40 \mathrm{~nm}$ & - & $\sim 30 / 10 \mathrm{~nm}$ & $30 / 6 \mathrm{~nm}$ \\
\hline$L_{\mathrm{g}}$ & $12 \mathrm{~nm}$ & - & $90 \mathrm{~nm}$ & $12 \mathrm{~nm}$ & $45 \mathrm{~nm}$ & $25.8 \mathrm{~nm}$ \\
\hline$S S(\mathrm{mV} / \mathrm{dec})$ & $75(\mathrm{~N}) / 85(\mathrm{P})$ & $65(\mathrm{~N}) / 67(\mathrm{P})$ & $140(\mathrm{~N}) / 130(\mathrm{P})$ & $73(\mathrm{~N}) / 74(\mathrm{P})$ & $64(\mathrm{~N})$ & $71.2(\mathrm{~N}) / 78.7(\mathrm{P})$ \\
\hline$D I B L(\mathrm{mV} / \mathrm{V})$ & $32(\mathrm{~N}) / 24(\mathrm{P})$ & $20(\mathrm{~N}) / 24(\mathrm{P})$ & - & $32(\mathrm{~N}) / 35(\mathrm{P})$ & $10(\mathrm{~N})$ & $9(\mathrm{~N}) / 22(\mathrm{P})$ \\
\hline$I_{\mathrm{ON}} / I_{\mathrm{OFF}}$ & $\sim 10^{4}$ & $\sim 10^{5}$ & $\sim 10^{4}$ & $\sim 10^{6}$ & $\sim 10^{5}$ & $3.15 \times 10^{5}$ \\
\hline
\end{tabular}

\section{Conclusions}

In summary, the optimization of NS release process of stacked GAA Si NS devices was carried out and achieved optimal GAA Si NS channels. An over 100:1 selective wet-etch ratio of GeSi to Si layer is achieved for GeSi/Si stack samples with different GeSi thickness and annealing temperatures $\left(\leq 900^{\circ} \mathrm{C}\right)$. Furthermore, the influence of the GP doping in Si sub-fin region to improve electrical characteristics of the devices was extensively investigated by experiments and simulations. The subthreshold characteristics of $n$-type devices are significantly improved with the increase of GP doping doses. However, the $p$-type devices were first improved and then deteriorated with the increase of GP doping doses which demonstrates better electrical characteristics with the GP doping concentrations of $1 \times 10^{18} \mathrm{~cm}^{-3}$. Furthermore, 4-layer stacked GAA Si NS channels with 6-nm-thick and 30-nm-width were firstly fabricated on bulk substrate, and the performance of the stacked GAA Si NS devices was greatly improved by the optimization of suppression of parasitic channels and device's structure, which achieved a larger $I_{\mathrm{ON}} / I_{\mathrm{OFF}}$ ratio and a smaller values of SSs and DIBLs. Therefore, the optimization of the fabricated stacked GAA Si NS devices approaches and simulated results provide a good guide for its potential mass application in future.

Author Contributions: Conceptualization, Q.Z., Z.Z., and H.Y. (Huaxiang Yin); epitaxy growth GeSi/Si stacks, G.W., H.H.R., and Z.K.; dry etch, J.L. (Junjie Li); E-beam lithography, X.H.; atomic layer deposition of high-k metal gate, J.X. and H.Y. (Hong Yang); source and drain contacts, J.L. (Jun Luo); process integration, Q.Z., R.X., J.Y., and J.T.; simulation, J.G., L.C., and Z.W.; characterization, G.X. and S.M.; data curation, Q.Z., J.G., L.C., and J.T.; writing-original draft, Q.Z., Z.Z., H.Y. (Huaxiang Yin), and J.G.; writing - review and editing, H.Y. (Huaxiang Yin) and Q.Z. All authors have read and agreed to the published version of the manuscript.

Funding: This work was supported in part by the National Key Project of Science and Technology of China under grant 2017ZX02315001-001and 2017ZX02301007-001, in part by the Science and Technology program of Beijing Municipal Science and Technology Commission under grants Z201100006820084 and Z201100004220001, the Youth Innovation Promotion Association, Chinese Academy of Sciences under grant Y9YQ01R004 and Y2020037, the National Natural Science Foundation of China under grants 61904194 and 6187032253, and in part by the Opening Project of Key Laboratory of Microelectronic Devices and Integrated Technology, Institute of Microelectronics, Chinese Academy of Sciences under Grant E0YS01X001 and E0290X03.

Data Availability Statement: The data presented in this study are available on request from the corresponding authors.

Acknowledgments: Thank you to Yongliang Li and Yongkui Zhang for results discussion; Qiuxia Xu and Hailing Tu for supervision; Junfeng Li for project administration; Wenwu Wang and Tianchun Ye for funding acquisition; and the Integrated Circuit Advanced Process Center (ICAC) at the Institute of Microelectronics of the Chinese Academy of Sciences for the devices fabricated on the advanced $200 \mathrm{~mm}$ CMOS platform. 
Conflicts of Interest: The authors declare no conflict of interest.

\section{References}

1. Natarajan, S.; Agostinelli, M.; Akbar, S.; Bost, M.; Bowonder, A.; Chikarmane, V.; Chouksey, S.; Dasgupta, A.; Fischer, K.; Fu, Q.; et al. A $14 \mathrm{~nm}$ logic technology featuring 2 nd-generation FinFET, air-gapped interconnects, self-aligned double patterning and a $0.0588 \mu \mathrm{m} 2$ SRAM cell size. In Proceedings of the 2014 IEEE International Electron Devices Meeting, San Francisco, CA, USA, 15-17 December 2014; pp. 71-73.

2. Cho, H.-J.; Oh, H.S.; Nam, K.J.; Kim, Y.H.; Yeo, K.H.; Kim, W.D.; Chung, Y.S.; Nam, Y.S.; Kim, S.M.; Kwon, W.H.; et al. Si FinFET based $10 \mathrm{~nm}$ technology with multi Vt gate stack for low power and high performance applications. In Proceedings of the 2016 IEEE Symposium on VLSI Technology, Honolulu, HI, USA, 14-16 June 2016; pp. 1-2.

3. Cheng, K.; Seo, S.; Faltermeier, J.; Lu, D.; Standaert, T.; Ok, I.; Khakifirooz, A.; Vega, R.; Levin, T.; Li, J.; et al. Bottom oxidation through STI (BOTS) - A novel approach toabricate dielectric isolated FinFETs on bulk substrates. In Proceedings of the 2014 Symposium on VLSI Technology (VLSI-Technology): Digest of Technical Papers, Honolulu, HI, USA, 9-12 June 2014; pp. 1-2.

4. Zhang, Q.; Yin, H.; Luo, J.; Yang, H.; Meng, L.; Li, Y.; Wu, Z.; Zhang, Y.; Zhang, Y.; Qin, C.; et al. FOI FinFET with ultra-low parasitic resistance enabled by fully metallic source and drain formation on isolated bulk-fin. In Proceedings of the 2016 IEEE International Electron Devices Meeting (IEDM), San Francisco, CA, USA, 3-7 December 2016; pp. $452-455$.

5. Radamson, H.H.; He, X.; Zhang, Q.; Liu, J.; Cui, H.; Xiang, J.; Kong, Z.; Xiong, W.; Li, J.; Gao, J.; et al. Miniaturization of CMOS. Micromachines 2019, 10, 293. [CrossRef] [PubMed]

6. Shirazi, S.G.; Karimi, G.R.; Mirzakuchaki, S. GAA CNT TFETs Structural Engineering: A Higher ON Current, Lower Ambipolarity. IEEE Trans. Electron Devices 2019, 66, 2822-2830. [CrossRef]

7. Yin, X.; Xie, L.; Ai, X.Z.; Zhang, Y.B.; Jia, K.P.; Wu, Z.H.; Ma, X.L.; Zhang, Q.Z.; Mao, S.J.; Xiang, J.J.; et al. Vertical sandwich gate-all-around field-effect transistors with self-aligned high-k metal gates and small effective-gate-length variation. IEEE Electron Device Lett. 2019, 41, 8-11. [CrossRef]

8. Song, Y.; Zhou, H.; Xu, Q.; Niu, J.; Yan, J.; Zhao, C.; Zhong, H. High-Performance Silicon Nanowire Gate-All-Around nMOSFETs Fabricated on BulkSubstrate Using CMOS-Compatible Process. IEEE Electron Device Lett. 2010, 31, 1377-1379. [CrossRef]

9. Li, M.; Fan, J.; Xu, X.; Huang, R. Investigation on electrostatic discharge robustness of gate-all-around silicon nanowire transistors combined with thermal analysis. IEEE Electron Device Lett. 2017, 38, 1653-1656. [CrossRef]

10. Huang, N.; Liu, W.; Li, Q.; Bai, W.; Yang, T. Investigation and optimization of electrical and thermal performance for 5-nm gaa vertically stacked nanowire fets. Microelectron. J. 2019, 95, 104679. [CrossRef]

11. Bae, G.; Bae, D.-I.; Kang, M.; Hwang, S.M.; Kim, S.S.; Seo, B.; Kwon, T.Y.; Lee, T.J.; Moon, C.; Choi, Y.M.; et al. 3nm GAA Technology featuring Multi-Bridge-Channel FET for Low Power and High Performance Applications. In Proceedings of the 2018 IEEE International Electron Devices Meeting (IEDM), San Francisco, CA, USA, 1-5 December 2018; pp. 28.7.1-28.7.4.

12. Chu, C.; Wu, K.; Luo, G.L.; Chen, B.Y.; Chen, S.H.; Wu, W.F.; Yeh, W.K. Stacked ge-nanosheet gaa fets fabricated by ge/si multilayer epitaxy. IEEE Electron Device Lett. 2018, 39, 1133-1136. [CrossRef]

13. Zhang, J.; Frougier, J.; Greene, A.; Miao, X.; Vega, R.; Montanini, P.; Durfee, C.; Gaul, A.; Pancharatnam, S.; Adams, C.; et al. Full Bottom Dielectric Isolation to Enable Stacked Nanosheet Transistor for Low Power and High Performance Applications. In Proceedings of the 2019 IEEE International Electron Devices Meeting (IEDM), San Francisco, CA, USA, 7-11 December 2019; pp. 11.6.1-11.6.4.

14. Barraud, S.; Previtali, B.; Vizioz, C.; Hartmann, J.M.; Sturm, J.; Lassarre, J.; Perrot, C.; Rodriguez, P.; Loup, V.; Magalhaes-Lucas, A.; et al. 7-Levels-Stacked Nanosheet GAA Transistors for High Performance Computing. In Proceedings of the 2020 IEEE Symposium on VLSI Technology, Honolulu, HI, USA, 16-19 June 2020; pp. 1-2.

15. Zhang, Z.; Xu, G.; Zhang, Q.; Hou, Z.; Li, J.; Kong, Z.; Zhang, Y.K.; Xiang, J.J.; Xu, Q.X.; Wu, Z.H.; et al. Finfet with improved subthreshold swing and drain current using $3 \mathrm{~nm}$ ferroelectric $\mathrm{hf}_{0.5} \mathrm{Zr}_{0.5} \mathrm{O}_{2}$. IEEE Electron Device Lett. 2019, 40, 367-370. [CrossRef]

16. Xu, M.; Zhu, H.; Zhang, Y.; Xu, Q.; Zhang, Y.; Qin, C.; Zhang, Q.Z.; Yin, H.X.; Xu, G.B.; Chen, S.; et al. Two methods of tuning threshold voltage of bulk finfets with replacement high-k metal-gate stacks. Solid-State Electron. 2017, 129, 52-60. [CrossRef]

17. Mertens, H.; Ritzenthaler, R.; Chasin, A.; Schram, T.; Kunnen, E.; Hikavyy, A.; Ragnarsson, L.-Å.; Dekkers, H.; Hopf, T.; Wostyn, K.; et al. Vertically stacked gate-all-around Si nanowire CMOS transistors with dual work function metal gates. In Proceedings of the 2016 IEEE International Electron Devices Meeting (IEDM), San Francisco, CA, USA, 3-7 December 2016; pp. 524-527.

18. Zhang, Q.; Tu, H.; Gu, S.; Zhang, Z.; Wang, G.; Wei, F.; Ma, T.D.; Zhao, H.B.; Wei, Q.H.; Yin, H.X.; et al. Influence of rapid thermal annealing on ge-si interdiffusion in epitaxial multilayer ge $0.3 \mathrm{si} 0.7 /$ si superlattices with various gesi thicknesses. ECS J. Solid State Sci. Technol. 2018, 7, 671-676. [CrossRef]

19. Li, C.; Zhu, H.; Zhang, Y.; Yin, X.; Jia, K.; Li, J.; Wang, G.L.; Kong, Z.Z.; Du, A.Y.; Yang, T.Z.; et al. Selective Digital Etching of Silicon-Germanium Using Nitric and Hydrofluoric Acids. ACS Appl. Mater. Interfaces 2020, 12, 48170-48178. [CrossRef] [PubMed]

20. Li, J.; Li, Y.; Zhou, N.; Xiong, W.; Wang, G.; Zhang, Q.; Du, A.; Gao, J.; Kong, Z.; Lin, H.; et al. Study of Silicon Nitride Inner Spacer Formation in Process of Gate-all-around Nano-Transistors. Nanomaterials 2020, 10, 793. [CrossRef] [PubMed]

21. Su, Y.; Lai, J.; Sun, L. Investigation of self-heating effects in vacuum gate dielectric gate-all-around vertically stacked silicon nanowire field effect transistors. IEEE Trans. Electron Devices 2020, 67, 4085-4091. [CrossRef] 


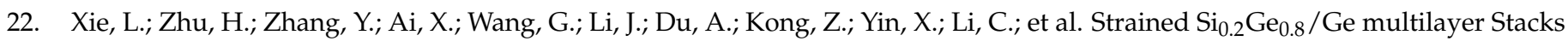
Epitaxially Grown on a Low-/High-Temperature Ge Buffer Layer and Selective Wet-Etching of Germanium. Nanomaterials 2020, 10, 1715. [CrossRef] [PubMed]

23. Liu, H.; Li, Y.; Cheng, X.; Zan, Y.; Lin, H.X.; Kong, Z.Z.; Zhong, Z.Y.; Li, Y.; Wang, H.Y.; Xu, G.B.; et al. Fabrication and selective wet etching of si0.2ge0.8/ge multilayer for si0.2ge0.8 channel gate-all-around mosfets. Mater. Sci. Semicond. Process. 2020, 121, 105397. [CrossRef]

24. Hu, R.; Xu, S.; Wang, J.; Shi, Y.; Yu, L. Unprecedented uniform 3d growth integration of 10-layer stacked si nanowires on tightly confined sidewall grooves. Nano Lett. 2020, 20, 10. [CrossRef] [PubMed]

25. Zhang, Q.; Tu, H.; Zhang, Z.; Li, J.; Wei, F.; Wang, G.L.; Han, J.H.; Zhao, H.B.; Zhang, Y.K.; Li, Y.L.; et al. Optimization of zero-level interlayer dielectric materials for gate-all-around silicon nanowire channel fabrication in a replacement metal gate process. Mater. Sci. Semicond. Process. 2021, 121, 105434. [CrossRef]

26. Sung, P.J.; Chang, S.W.; Kao, K.H.; Wu, C.T.; Chao, T.S. Fabrication of vertically stacked nanosheet junctionless field-effect transistors and applications for the cmos and cfet inverters. IEEE Trans. Electron Devices 2020, 67, 9. [CrossRef]

27. Kim, S.; Kim, M.; Ryu, D.; Lee, K.; Park, B.G. Investigation of electrical characteristic behavior induced by channel-release process in stacked nanosheet gate-all-around mosfets. IEEE Trans. Electron Devices 2020, 67, 6. [CrossRef]

28. International Roadmap for Devices and systems 2017 Edition More Moore. Available online: https://irds.ieee.org/images / files/ pdf/2017/2017IRDS_MM.pdf (accessed on 30 December 2018).

29. Yao, J.X.; Li, J.; Luo, K.; Yu, J.H.; Zhang, Q.Z.; Hou, Z.H.; Gu, J.; Yang, W.; Wu, Z.H.; Yin, H.X.; et al. Physical insights on quantum confinement and carrier mobility in si, sige, ge gate-all-around nsfet for $5 \mathrm{~nm}$ technology node. IEEE J. Electron Devices Soc. 2018, 6, 841-848. [CrossRef]

30. Loubet, N.; Hook, T.; Montanini, P.; Yeung, C.W.; Kanakasabapathy, S.; Guillom, M.; Yamashita, T.; Zhang, H.; Miao, X.; Wang, J.; et al. Stacked nanosheet gate-all-around transistor to enable scaling beyond FinFET. In Proceedings of the 2017 Symposium on VLSI Technology, Kyoto, Japan, 5-8 June 2017; pp. T230-T231. 